\title{
MASS EXTINCTIONS, COMET IMPACTS, AND THE GALAXY
}

\author{
MICHAEL R. RAMPINO \\ NASA, Goddard Institute for Space Studies, New York, New York, 10025 USA, \\ and Earth 8 Environmental Science Program, New York University, New York, \\ New York, 10003 USA \\ AND \\ RICHARD B. STOTHERS \\ NASA, Goddard Institute for Space Studies, New York, New York, 10025 USA
}

\begin{abstract}
The hypothesis relating mass extinctions of life on Earth to impacts of comets whose flux is partly modulated by the dynamics of the Milky Way Galaxy contains a number of postulates that can be tested by geologic evidence and statistical analyses. In an increasing number of cases, geologic evidence for impact (widespread impact debris and/or large impact craters) is found at times of mass extinction events, and the record of dated impact craters has been found to show a significant corrclation with mass extinctions. Statistical analyses suggest that mass extinction events exhibit a periodic component of about 26 to $30 \mathrm{Myr}$, and periodicities of $30 \pm 0.5 \mathrm{Myr}$ and $35 \pm 2 \mathrm{Myr}$ have been extracted from sets of well-dated impact craters. The evidence is consistent with periodic or quasi-periodic showers of impactors, probably Oort Cloud comets, with an approximately 30Myr cycle. The best explanation for these proposed quasi-periodic comet showers involves the Sun's vertical oscillation through the galactic disk, which may have a similar cycle time between crossings of the galactic plane.
\end{abstract}

\section{INTRODUCTION: IMPACTS AND MASS EXTINCTIONS}

Numerous studies have now established that the mass extinction of life at the end of the Cretaceous Period (65 Myr ago), the so-called Cretaceous/Tertiary (K/T) boundary, was the result of the impact of an asteroid or comet $\sim 10 \mathrm{~km}$ in diameter which created the $\geq 200 \mathrm{~km}$ diameter Chicxulub impact structure in the present Yucatán region of Mexico (Alvarez, 1986; Hildebrand et al., 1995). Such an impact (releasing $>10^{24} \mathrm{~J}$ or $10^{8} \mathrm{Mt}$. TNT equivalent) is calculated to cause a global catastrophe of enormous proportions, primarily related to dense clouds of fine ejecta, production of nitric oxides and acid rain, and smoke clouds from fires triggered by atmospheric re-entry of ejecta (Toon et al., 1994). Other "target-sensitive" effects include enhanced grenhouse effect from atmospheric water vapor in an ocean impact, $\mathrm{CO}_{2}$ released by impact into carbonate rocks, and cooling and acid rain from large amounts of sulfuric acid acrosols derived from calcium sulfate target rocks (Pope et al., 1994; Toon et al., 1994). If flood basalt eruptions are impact triggered (Rampino and Stothers, 1988), then volcanic perturbations of climate might also be expected.

Astronomical observations of Earth-crossing asteroids and comets and the cratering record of the inner planets allow estimates of the expected mean times between collisions of objects of various sizes with the planet (Shoemaker et al., 1990). On the large-diameter end of the size spectrum, these data predict that in an $\sim 100$ Myr period, the Earth should be hit by several asteroids and comets larger than a few $\mathrm{km}$ in diameter (these release $\sim 10^{23} \mathrm{~J}$ or $10^{7} \mathrm{Mt}$ ) and perhaps one $\sim 10 \mathrm{~km}$ diameter $\left(\sim 10^{24} \mathrm{~J}, 10^{8} \mathrm{Mt}\right.$ ) object (most likely cometary) (Shoemaker et al., 1990).

The likelihood that a $10^{8} \mathrm{Mt}$ event, would have considerably greater global envirommental impact. than a $10^{7}$ Mt event (Melosh et al., 1990; Toon et al., 1994), leads to the prediction of $\sim 5$ major mass extinctions, and about $25 \pm 5$ less severe pulses of extinction during the Phancrozoic Eon (the 


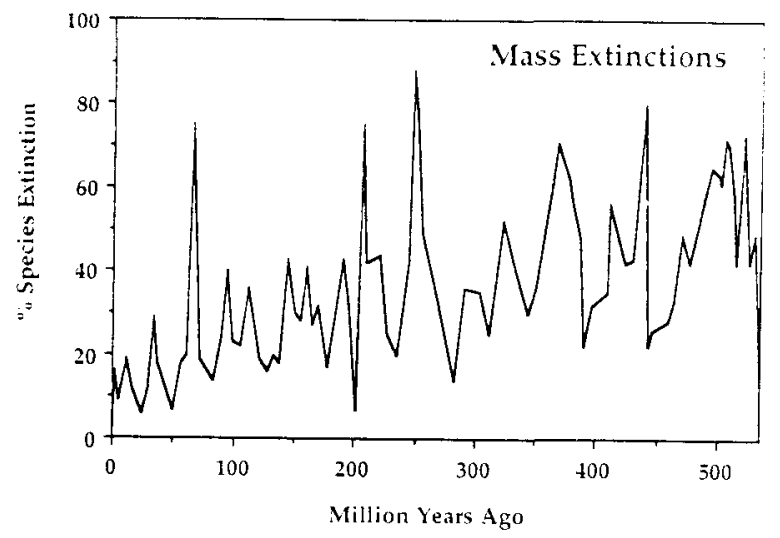

Figure 1. Percent extinction of marine species per geologic stage (or substage) during the Phanerozoic (data from Sepkoski, 1995).

TABLE 1. Large Dated Impact Craters (Grieve and Pesonen, 1996) with updates and Associated Extinctions (Sepkoski, 1995)

\begin{tabular}{|l|cllc|}
\hline Name & Diameter $(\mathrm{km})$ & Age (Myr) & Extinction & \% Species \\
\hline Popigai & 100 & $35.7 \pm 0.8$ & Late Eocene & 30 \\
Chesapeake & 90 & $35.2 \pm 0.3$ & Late Eocene & - \\
Chicxulub & $200 ?$ & $65.2 \pm 0.4$ & K/T & 76 \\
Morokweng & 100 & $145 \pm 3$ & J/K & 42 \\
Manicouagan & 100 & $214 \pm 1$ & Late Triassic & 76 \\
Puchezh-Katunki & 80 & $220 \pm 10$ & Carnian & 42 \\
\hline
\end{tabular}

last $540 \mathrm{Myr}$ ). This agrees surprisingly well with the record of extinctions for that interval, which clearly shows 5 major mass extinctions and $\sim 20$ less severe extinction pulses (Fig. 1).

Although the severity of impact-induced mass extinctions would probably depend on a number of variables (e.g. ambient climate conditions, susceptibility of fauna and flora, site of impact, etc.), the size and energy of the impactor is likely to be the most important factor. A theoretical "kill curve" relating mass extinctions and impacts (Fig. 2) has been proposed (Raup, 1992), and this curve can be compared with data representing specific large (> $80 \mathrm{~km}$ diameter) known impact craters with well-defined ages that overlap the ages of mass-extinction boundaries (when the full dating uncertainties in both are taken into consideration) (Table 1). We note that no other large, well-dated Phanerozoic craters are known. The observed points in Fig. 2 agree with the predicted curve within the envelope of error permitted by the geologic data, supporting at least a first-order relationship between large impacts and mass extinctions.

\section{IS THERE A CORRELATION BETWEEN IMPACTS AND MASS EXTINC- TION EVENTS?}

The discovery of the $\mathrm{K} / \mathrm{T}$ impact layer prompted the search for impact signatures at other geological boundaries. Among the materials considered diagnostic of impact are shocked minerals (including shocked quartz, stishovite, shocked zircons, etc.), impact glass (microtektites/tektites), microspherules with structures indicating high-temperature origin, and Ni-rich spinels. Shocked minerals and tektite glass are quite rare in the geologic record, and yet these materials have been reported in stratigraphic horizons close to the times of six recorded extinction pulses (Table 2) (Rampino et al., 1997; Grieve, 1997). A recent preliminary report of shocked quartz from the Permian/Triassic boundary ( $248 \mathrm{Myr}$ ) in Antarctica and Australia (Retallack et al., 1996) is still 


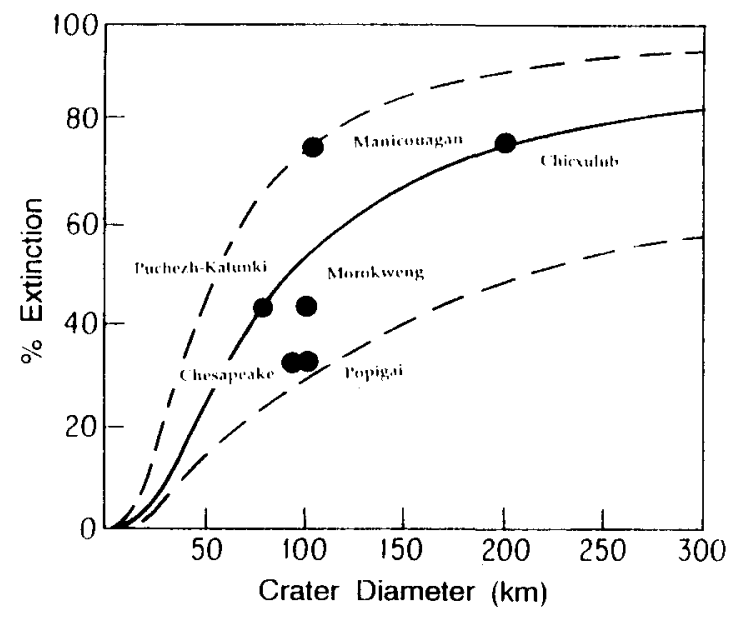

Figure 2. Kill curve for Phanerozoic marine species, with estimated error bars, plotted against estimated size of impact craters associated with extinctions of various magnitudes (assuming that the two are related) (Raup, 1992) Largest well-dated impact craters with ages overlapping mass extinction times are plotted for comparison (Table 1).

unconfirmed.

Seven of the $\sim 25$ extinction peaks in Fig. 1 [Pliocene (2.3 Myr), Late Eocene (36 Myr), Cretaceous/Tertiary (65 Myr), Jurassic/Cretaceous (144 Myr), Late Triassic (210 Myr), Late Devonian (365 Myr), and possibly the Proterozoic/Cambrian (540 Myr) boundary] are associated with large impact craters and/or some form of stratigraphic evidence of impacts (Tables 1 and 2). Several other extinction events are associated with "possible" evidence of impact consisting of iridium concentrations somewhat elevated with respect to background values; see tables in (Rampino et al., 1997; Grieve, 1997).

The chances of accidentally matching a time series consisting of 24 extinction events (with the age error bars in this case taken as the difference in ages given by recent published time scales), with a time series consisting of the seven times of diagnostic stratigraphic evidence of impacts is $<10^{-7}$. Studies thus far indicate that iridium anomalies above background and shocked quartz are rare in the geologic record, also suggesting that correlation of mass-extinction events and evidence of large impacts is very unlikely to be accidental.

Recently, Matsumoto and Kubotani (1996) showed a statistically significant correlation between the record of impact cratering of the last $250 \mathrm{Myr}$ and the record of mass extinctions. Stothers (1993) had earlicr found a significant correlation between impact crater ages and stratigraphic stage, boundaries that are defined by faunal changes during the Cenozoic Era (the last $65 \mathrm{Myr}$ ). These studies support a general correlation between impacts and extinctions of various magnitudes from significant mass extinctions ( $\geq 10^{8}$ to $10^{7} \mathrm{Mt}$ impacts) to the less severe faunal turnover events that mark stratigraphic stage boundaries $\left(\sim 10^{6}\right.$ to $10^{7} \mathrm{Mt}$ impacts $)$.

TABLE 2. Stratigraphic Evidence of Impacts at or near Extinction Boundaries (for references see text)

\begin{tabular}{|l|l|}
\hline Age & Evidence \\
\hline Pliocene (2.3 Myr) & Microkrystites, microtektites? \\
Late Eocene (36 Myr) & Microtektites (multiple), tektites, microspherules, shocked quartz \\
Cretaceous/Tertiary (65 Myr) & Microtektites, tektites, shocked minerals, stishovite, \\
& Ni-rich spinels, iridium \\
Jurassic/Cretaceous (142 Myr) & Shocked quartz \\
Late Triassic (210 Myr) & Shocked quartz (multiple?) \\
Late Devonian (368-365 Myr) & Microtektites (multiple) \\
\hline
\end{tabular}




\section{ARE MASS EXTINCTIONS AND IMPACTS PERIODIC?}

The mean occurrence rate of the 24 extinction pulses shown in Fig. 1 is one every $\sim 23$ Myr. Raup and Sepkoski $(1984,1986)$ reported a statistically significant 26.4 Myr periodicity in extinction time series for the last $250 \mathrm{Myr}$, and a secondary periodicity of $30 \mathrm{Myr}$. Periods of $\sim 26$ to $31 \mathrm{Myr}$ have been derived using various subsets of extinction events (family and genus levels), different geologic time scales, and various methods of time-series analysis, and Rampino and Stothers (1986) reported detection of an $\sim 29 \mathrm{Myr}$ component in the record of extinctions of non-marine vertebrates. The interpretation of these results has been a subject of considerable debate (e.g., Rampino and Haggerty, 1996). The detection of a periodic component in extinctions does not imply either that the record contains a strict periodicity, or that all extinction events follow a 26-Myr timescale; the extinction record might be a mixture of periodic and random events.

Fourier analysis of an extended record of 21 extinctions going back to about $515 \mathrm{Myr}$, more than doubling the length of the original time series, resulted in a spectrum with the highest peak at 27.3 Myr (Rampino and Haggerty, 1996). Fourier analyses on a series of truncated extinction time series starting from 0 to $540 \mathrm{Myr}$, and subtracting one extinction at a time back to $253 \mathrm{Myr}$ produced a stable peak between 26.5 and 27.3 Myr which remained the dominant feature in the spectra (Rampino and Haggerty, 1996).

The impact-crater record has been interpreted as showing pulses at about $1,35,65$, and $95 \mathrm{Myr}$ ago (Shoemaker and Wolfe, 1986; Hut et al., 1987). Time-series analyses of terrestrial impact craters have provided some evidence of a possible 28 to 32 million year periodicity in impacts (Rampino and Stothers, 1984a, 1984b, 1986; Alvarez and Muller, 1984; Shoemaker and Wolfe, 1986; Yabushita, $1991,1992,1996 \mathrm{a}, 1996 \mathrm{~b})$, and have been interpreted as suggesting that the impact record may be a mixture of periodic and random events (Trefil and Raup, 1987; Heisler and Tremaine, 1989; Fogg, 1989). Several studies have concluded that, considering the problems in dating, the observed differences in the formal periodicity detected in impact craters and mass extinctions are to be expected (Stothers, 1988, 1989; Fogg, 1989). Critics have argued, however, that the number of welldated craters is still too small to extract a consistent statistically significant periodicity (Grieve and Shoemaker, 1994; Grieve and Pesonen, 1996).

\section{A GALACTIC FORCING FOR PERIODIC IMPACTS AND EXTINCTIONS?}

In the galactic models, the flux of long-period comets (comet showers) is modulated by the periodic passage of the solar system through the central plane of the Milky Way Galaxy (Rampino and Stothers, 1984a; Matese et al., 1995). The original model (Rampino and Stothers, 1984a) proposed that the probability of encounters with molecular clouds that would perturb the Oort comet cloud causing comet showers would be modulated by the Sun's oscillation about the galactic disk. The rather flat distribution of clouds around the galactic plane suggests that an encounter would be more likely as the Sun passed through the plane region, and hence the encounters would be quasiperiodic, with a period equal to the time between plane crossings (Rampino and Stothers, 1986). Extensive numerical simulations have shown that this effect should be detectable in the terrestrial record of impact cratering with at least a $50 \%$ a priori probability (Stothers, 1985).

Matese et al. (1995) suggested that time modulation of the flux of new Jupiter-dominated Oort cloud comets could come from gravitational perturbations of the comet cloud by the adiabatically varying galactic tides during the in-and-out of plane oscillation; see also (Scalo and Smoluchowski, 1984). One remaining uncertainty is the most likely cycle time. The cycle time and modulation depend critically on the mass distribution in the galactic disk, particularly the distribution of dark matter in the disk. As Matese et al. (1995) have shown: (1) If there is no dark matter in the disk, then the mean plane crossing period is $\sim 44 \mathrm{Myr}$, and the peak-to-trough ratio in the comet flux is about 2.5 to 1. (2) If the current best estimate of local disk density is used, then the mean plane crossing period is $\sim 33 \mathrm{Myr}$, and the peak-to-trough flux ratio is about 4 to 1 . (3) If the estimated errors of the various recent estimates in the disk matter density are considered, then the period could be as short as about $28 \mathrm{Myr}$, with a comet flux ratio of 4 to 1 . The issue of dark disk matter is still unsettled (Gould et al., 1996), and if there is substantial dark matter, the most likely remaining source may be very cold molecular clouds (Stothers, 1984; Lequeux et al., 1993).

Using the current best estimate of disk mass density, Matese et al. (1995) found that the most recent times of peak comet flux were $\sim 1 \mathrm{Myr}$ in the future, and also about 31,65 , and $98 \mathrm{Myr}$ 
TABLE 3. Results of Spectral Analyses of Impact Crater Ages

\begin{tabular}{|l|l|l|c|c|}
\hline Smallest Diameter & $N$ & Phase & Highest Peak (Myr) & Second highest Peak (Myr) \\
\hline $5 \mathrm{~km}$ & 31 & Free & 30 & 35 \\
& & Fixed & 30 & 35 \\
$35 \mathrm{~km}$ & \multirow{3}{*}{11} & Free & 35 & None \\
& & Fixed & 35 & None \\
& \multirow{3}{*}{5} & Free & 36 & 29 \\
& & Fixed & 36 & 30 \\
\hline
\end{tabular}

ago, and that the cycle interval varied from 29.5 to $34.2 \mathrm{Myr}$ over a $350 \mathrm{Myr}$ run of their galactic model. Peak flux times lag the times of galactic plane crossing by about $1 \mathrm{Myr}$. Shoemaker et al. (1990) maintained that the largest impactors should be comets, and hence the largest craters should preferentially show the galactic modulation. Such pulses of increased comet flux would explain the stepped nature of some extinction events, clusters of similar-age craters, and multiple impact layers seen in the geologic record. Both galactic models must assume that shower conets come from the Oort cloud, in numbers moderately greater than background comets and asteroids (especially comets from the Kuiper belt).

In order to test the consistency of the latest cratering and extinction data with the galactic models, we performed a series of new linear spectral analyses on these data. Using the 11 mass extinctions over the last $250 \mathrm{Myr}$, we have performed the time-series analyses either allowing the phase to be a free parameter, or with fixed phasing, in which case we only used trial starting epochs in the range $\pm 1 \mathrm{Myr}$ (the time of most recent galactic plane crossing). When the phase is allowed to vary, only one high spectral peak at $27 \mathrm{Myr}$ is apparent. When the phase is fixed at the present time, the highest peak shifts to $28 \mathrm{Myr}$, but two somewhat smaller peaks at 32 and 35 Myr become considerably more prominent. These results suggest that although the average time interval between these mass extinctions is $25 \mathrm{Myr}$, the spacing is actually somewhat irregular, and so one need not accept $27 \mathrm{Myr}$ as the "correct" period.

To test the crater record, we performed linear time-series analyses on sets of various size craters using the impact crater list of (Grieve and Pesonen, 1996) containing 32 craters, to which we added the Chesapeake crater $(90 \mathrm{~km}, 35.2 \pm 0.3 \mathrm{Myr})$, the Mjölnir crater $(40 \mathrm{~km}, 142 \pm 2.6 \mathrm{Myr})$, the Morokweng crater (>100 km, $145 \pm 3 \mathrm{Myr}$ ), and a correction for the ages of the Rochechouart $(214 \pm 8 \mathrm{Myr})$ and Popigai (35.7 $\pm 0.8 \mathrm{Myr}$ ) craters (Grieve, 1996, 1997; Rampino et al., 1997). In all cases, the upper age limit was taken to be $250 \mathrm{Myr}$, and only those ages with errors of less than $\pm 10 \mathrm{Myr}$ were used. Table 3 shows the results for the best-fitting periods obtained.

As Table 3 shows, we detected two peaks, a narrow peak at $30 \pm 0.5 \mathrm{Myr}$ and a broader peak at $35 \pm 2 \mathrm{Myr}$. In a study designed to test the effects of the errors in the ages of the craters on the periods detected, Stothers (1988) showed that the errors alone were capable of shifting the dominant period between 30 and $35 \mathrm{Myr}$. We note, however, that the periods detected in the mass extinction and cratering records are statistically significant (at the 5\% level) only when they are treated as periods known a priori.

Our studies show that the geologic data on mass extinctions and evidence of large impacts are consistent with a quasi-periodic modulation of the flux of Oort cloud comets with a mean period of about 30 to $36 \mathrm{Myr}$, as predicted by curront galactic models. Further astronomical and geological studies should help to refine both the astronomical cycle time and the periodicity detectable in the geologic record.

\section{References}

Alvarez, W. 1986. Toward a. Theory of Impact Crises. Eos, Transactions of the American Guphysicul Union 67. $649-658$.

Alvarez, W. and R.A. Muller 1984. Evidence From Crater Ages for Periodic Impacts on the Earth. Nature 308 , $718-720$.

Fogg, M.J. 1989. The Relevance of the Background Impact Flux to Cyclic: Impact/Mass Extinction Hypotheses. Icarus $79,382-395$. 
Gould, A., Bahcall, J.N. and C. Flynn 1996. Disk M Dwarf Luminosity Function from Hubble Space Telescope Star Counts. Astrophys. J. 465, 759-768.

Grieve, R.A.F. 1996. Chesapeake Bay and Other Terminal Eocene Impacts. Meteoritics and Planetary Science 31, $166-167$.

Grieve, R.A.F. 1997. Extraterrestrial Impact Events: The Record in the Rocks and the Stratigraphic Column. Palaeoclimatology, Palaeogeography, Palaeoecology 132, 5-23.

Grieve, R.A.F. and L.J. Pesonen 1996. Terrestrial Impact Craters: Their Spatial and Temporal Distribution and Impacting Bodies. Earth, Moon and Planets 72, 357-376.

Grieve, R.A.F. and E.M. Shoemaker 1994. The Record of Past Impacts on Earth. Hazards Due to Comets 89 Asteroids (T. Gehrels, Ed.), 417-462. Univ. of Arizona Press, Tucson.

Heisler, J. and S. Tremaine 1989. How Dating Uncertainties Affect the Detection of Periodicity in Extinctions and Craters. Icarus 77, 213-219.

Hildebrand, A.R., Pilkington, M., Connors, M., Ortiz-Aleman, C. and R.E. Chavez 1995. Size and Structure of the Chicxulub Crater Revealed by Horizontal Gravity Gradients and Cenotes. Nature 376, 415-417.

Hut, P., Alvarez, W., Elder, W.P., Hansen, T., Kauffman, E.G., Keller, G., Shoemaker, E.M. and P.R. Weissman 1987. Comet Showers as a Cause of Mass Extinctions. Nature 329, 118-126.

Lequeux, J., Allen, R.J. and S. Guilloteau 1993. CO Absorption in the Outer Galaxy, Abundant Cold Molecular Gas. Astronomy and Astrophysics 280, L23-L26.

Matese, J.J., Whitman, P.G., Innanen, K.A. and M.J. Valtonen 1995. Periodic Modulation of the Oort Cloud Comet Flux by the Adiabatically Changing Galactic Tide. Icarus 116, 255-268.

Matsumoto, M. and H. Kubotani 1996. A Statistical Test for Correlation Between Crater Formation Rate and Mass Extinctions. MNRAS 282, 1407-1412.

Melosh, H.J., Schneider, N.M., Zahnle, K.J. and D. Latham 1990. Ignition of Global Wildfires at the Cretaceous/Tertiary Boundary. Nature 343, 251-254.

Pope, K. O., Baines, K.H., Ocampo, A.C. and B.A. Ivanov 1994. Impact Winter and the Cretaceous/Tertiary Extinctions: Results of a Chicxulub Asteroid Impact Model. Earth and Planetary Science Letters 128, 719-725.

Rampino, M.R. and B.M. Haggerty 1996. The "Shiva Hypothesis": Impacts, Mass Extinctions, and the Galaxy. Earth, Moon and Planets 72, 441-460.

Rampino, M.R., Haggerty, B.M. and T.C. Pagano 1997. A Unified Theory of Impact Crises and Mass Extinctions: Quantitative Tests. Annals of the New York Academy of Sciences 822, 403-431.

Rampino, M.R. and R.B. Stothers 1984a. Terrestrial Mass Extinctions, Cometary Impacts and the Sun's Motion Perpendicular to the Galactic Plane. Nature 308, 709-712.

Rampino, M.R. and R.B. Stothers 1984b. Geological Rhythms and Cometary Impacts. Science 226, 1427-1431.

Rampino, M.R. and R.B. Stothers 1986. Geologic Periodicities and the Galaxy. The Galaxy and the Solar System (R. Smoluchowski, J. N. Bahcall and M. S. Matthews, Eds.), 241-259. Univ. of Arizona Press, Tucson.

Rampino, M.R. and R.B. Stothers 1988. Flood Basalt Volcanism During the Past 250 Million Years. Science 241, $663-668$

Raup, D.M. 1992. Large-Body Impact and Extinction in the Phanerozoic. Paleobiology 18, 80-88.

Raup, D.M. and J.J. Sepkoski, Jr. 1984. Periodicity of Extinctions in the Geologic Past. Proceedings of the National Academy of Sciences USA 81, 801-805.

Raup, D.M. and J.J. Sepkoski, Jr, 1986. Periodic Extinctions of Families and Genera. Science 231, 833-836.

Retallack, G.J., Seyedolali, A., Holser, W.T., Krinsley, D. and E.S. Krull 1996. Shocked Quartz at the Permian-Triassic Boundary in Australia and Antarctica. Geological Society of America Abstracts with Programs 28, A-368.

Scalo, J.M. and R. Smoluchowski 1984. Galactic Gravitational Shock and the Extinction of Species. Bulletin of the American Astronomical Society 16, 493-494.

Sepkoski, J.J., Jr. 1995. Patterns of Phanerozoic Extinction: A perspective From Global Data Bases. Global Events and Event Stratigraphy in the Phanerozoic (O.H. Walliser, Ed.), 35-51. Springer, Berlin.

Shoemaker, E.M. and R.F. Wolfe 1986. Mass Extinctions, Crater Ages, and Comet Showers. The Galaxy and the Solar System (R. Smoluchowski, J.N. Bahcall and M.S. Matthews, Eds.), 338-386. Univ. of Arizona Press, Tucson.

Shoemaker, E.M., Wolfe, R.F. and C.S. Shoemaker 1990. Asteroid and Comet Flux in the Neighborhood of Earth. Geological Society of America Special Paper 247, 155-170

Stothers, R.B. 1984. Mass Extinctions and Missing Matter. Nature 311, 17

Stothers, R.B. 1985. Terrestrial Record of the Solar System's Oscillation About the Galactic Plane. Nature 317, $338-341$.

Stothers, R.B. 1988. Structure of Oort's Comet Cloud Inferred From Terrestrial Impact Craters. Observatory 108, $1-9$

Stothers, R.B. 1989. Structure and Dating Errors in the Geologic Time Scale and Periodicity in Mass Extinctions. Geophysical Research Letters 16, 119-122.

Stothers, R.B. 1993. Impact Cratering at Geologic Stage Boundaries. Geophysical Research Letters 20, 887-890.

Toon, O.B., Zahnle, K., Turco, R.P. and C. Covey 1994. Environmental Perturbations Caused by Asteroid Impacts. Hazards due to Asteroids and Comets (T. Gehrels, Ed.), 791-826. Univ. of Arizona Press, Tucson.

Trefil, J.S. and D.M. Raup 1987. Numerical Simulations and the Problem of Periodicity in the Cratering Record. Earth and Planetary Science Letters 82, 159-164.

Yabushita, S. 1991. A Statistical Test for Periodicity Hypothesis in the Crater Formation Rate. MNRAS 250, 481-485.

Yabushita, S. 1992. Periodicity in the Crater Formation Rate and Implications for Astronomical Modeling. Dynamics and Evolution of Minor Bodies with Galactic and Geological Implications (S.V.M. Clube, S. Yabushita and J. Henrard, Eds.), 161-178. Kluwer, Dordrecht.

Yabushita, S. 1996a. Are Cratering and Probably Related Geological Records Periodic? Earth, Moon, and Planets 72, 343-356

Yabushita, S. 1996b. Statistical Tests of a Periodicity Hypothesis For Crater Formation Rate-II. MNRAS 279, 727732. 